\title{
Brain Activity Viewing Various Tobacco Health Warning Posters
}

$$
\text { ChingSung } \mathrm{Ho}^{1, \mathrm{a}} \text {, SihHuei Chen }{ }^{2, \mathrm{~b}} \text {, KangMing Chang }{ }^{3,4, * *}
$$

${ }^{1}$ Department of Long Term Care, National Quemoy University, Quemoy, 89250, Taiwan

${ }^{2}$ Biosignal Processing Lab, ASIA University, Taichung, 41354, Taiwan

${ }^{3}$ Department of Photonics and Communication Engineering, ASIA University, Taichung, 41354, Taiwan

${ }^{4}$ Department of Medical Research, China Medical University Hospital, China Medical University, Taichung, 40402, Taiwan

aemail: csho@nqu.edu.tw, bemail: abbykayq@yahoo.com.tw, **email: Correspondence should be addressed to changkm@asia.edu.tw

Keywords: Electroencephalography; Tobacco; Power; Coherence

\begin{abstract}
Adolescent smoking is an increasing issue in the world. To reduce the harm of tobacco products for adolescent, there is warning posters. There is seldom evidence of these warning posters. In this article, brain wave response is used to detect various tobacco health warning posters. Thirty-one male high school students are enrolled. Five typical posters are evaluated, diseased organs, pregnant woman, political and public figures, young idol, and basic textual declaration. Landscape image is also used as compare. Each poster lasted for 30 seconds. Brain wave is recorded. Brain wave power and coherence of 32 electrodes are estimated. There are significant differences among five figures by brain response on different brain areas.
\end{abstract}

\section{Introduction}

Tobacco products have become a crucial factor causing health hazards worldwide. Based on an estimation by the World Health Organization (WHO), tobacco product-caused deaths will reach 64 million and 83 million by 2015 and 2030, respectively [1]. In Taiwan, more than 18000 people die from smoking-related diseases annually [2]. The annual National Health Insurance (Taiwan) expenditures on smoking-related diseases are approximately US\$290-330 million [3]. According to statistics from 2008, 38.6\% of men and 4.8\% of women among people aged older than 18 years in Taiwan smoke; $27.2 \%$ of all families were exposed to secondhand smoke in the past week; and $26 \%$ of interviewees reported that they were exposed to smoking colleagues in the workplace during the past week [4]. Tobacco prevention must begin at a young age, and the current ages of smokers have progressively decreased. In Taiwan, those who smoked accounted for 7.8\% (10.3\% male and 4.9\% female students) of all junior high school students in 2008, and 8.0\% (11.2\% male and $4.2 \%$ female students) in 2010. However, a 2009 survey on the smoking behavior of high school students in Taiwan revealed that $14.8 \%$ of high school students in Taiwan $(19.6 \%$ male and $9.1 \%$ female students) were smokers, which slightly declined from 15.2\% in 2005 (21.1\% male and 8.5\% female students). Those who smoked for the first time before the age of 10 years accounted for $20.7 \%$ of high school student smokers. Providing smoking cessation education to students is urgent in both the legal aspects and practical demands [5].

Concerns for tobacco hazards can be advocated through public channels to alter or strengthen the positive attitudes of adolescents toward anti-tobacco lifestyles. In recent years, the Health Promotion Administration, Ministry of Health and Welfare, Executive Yuan (Taiwan) has progressively evaluated the effectiveness of annual media advocacies by using the respective performance evaluation indicators and instruments. Chang et al. [6] showed that (graphic) cigarette warning labels induced discomfort and fear in $60 \%$ of the student participants and were associated with health hazards in more than $90 \%$ of the student participants. Approximately $60 \%$ of the students were repelled by the cigarette warning labels, $80 \%$ of the student participants wanted to 
convince smokers to cease smoking because of the warning, and $50 \%$ of the student smokers expressed their intention to cease smoking because of the warning labels. The Taiwanese government has long advocated numerous anti-tobacco messages, and public organizations have individually produced tobacco prevention advocacy products (i.e., promotional materials, short films, and pamphlets). However, advocacy information lacked long-term planning and has been independent; thus, its influential powers are limited even though the public has been exposed to a large amount of relevant information. Consequently, these efforts failed to alter the attitudes and behavior of the public, and thus, integrating advocacy instruments in tobacco prevention is a crucial task.

To reduce the harm of tobacco products, several countries have promoted tobacco prevention efforts and prohibited indoor and workplace smoking. Tobacco health warnings was the topic on World No Tobacco Day in 2009, and the WHO indicated that cigarette warning labels facilitated peoples' recognition of the health risks caused by tobacco products and convinced people to cease smoking [7]. Studies have shown that cigarette warning labels can induce public attention, induce smokers to rethink about the health risks associated with smoking, and increase motives and intention to cease smoking more effectively than textual warnings [8, 9]. In addition, these labels effectively prevent smoking behaviors and reduce intention to smoke $[10,11]$. Currently, tobacco prevention in Taiwan primarily focuses on the concept of suppression as an advocacy direction. Typical cigarette warning labels consist of either celebrity warnings or unpleasant images to warn consumers of the harms of smoking. The warning effects of these labels on smokers have primarily been examined through questionnaire surveys. However, the practical effects and influence of the labels on the viewers remain unclear. Regarding the study of brain science, numerous papers have explored the influence of image stimulation in brainwaves. Through brainwaves or visual evoked potentials in coordination with information algorithms, emotional statuses can be detected from brain responses [12-13]. In addition, a study investigated the physical harms of smoking by using electroencephalography (EEG) [14]. Inhaling nicotine altered the brainwaves and evoked potentials [15]. Furthermore, smokers have poorer response inhibition than nonsmokers, based on a go/nogo task [16]. Thus, we focused on whether brainwaves can be used to determine altering brain activities after smokers viewed cigarette warning labels. In this study, we tested five common cigarette warning labels and compared them with neutral landscape images. The participants of this study were currently smoking high school students.

\section{Methods}

\subsection{Subject Information}

We sampled students from a vocational high school in Taichung City, Taiwan. All of the students were peers who participated in the school's smoking cessation class and were confirmed to have regular smoking habits. We recruited 31 male students (boys' class) whose ages ranged from 16-18 years (an average of $17.4 \mathrm{y}$ ). Personal and legal guardian consents were obtained from all of the samples before the experiment. The vision and color discrimination abilities of all of the participants were normal, and the participants were free from drug and alcohol use, head injuries, and nervous system diseases. The participants were instructed to shampoo their hair the day before testing to prevent interference with the EEG waves. We followed the process approved by the Institutional Review Board of Asia University (document number 10012006), and informed participants in advance regarding the experimental process, duration, and possible conditions (e.g., conductive pastes can adhere to the hair and cause discomfort). According to experimental requirements, we then requested the participants to complete their demographic data and five questionnaires. The questionnaires comprised knowledge on tobacco hazard, anti-tobacco self-efficacy, anti-tobacco attitude, smoking cessation motives, and nicotine addiction measurements. The results of the survey analysis are discussed in a separate paper. 


\subsection{Experimental Procedure and Data Analysis}

The brainwaves at resting states were recorded prior to the experiment. When the experiment began, data were recorded $30 \mathrm{~s}$ after the blank screen was displayed, which was followed by displaying five warning labels: (a) diseased organs, (b) a pregnant woman, (c) political and public figures, (d) young idol, and (e) basic textual declaration; and then (f) the landscape image (Figure 1). Each picture was displayed for $30 \mathrm{~s}$ with intermediate blank screens lasting $30 \mathrm{~s}$ each. The viewing sequence of the warning labels (randomly arranged) varied for each participant. We used the NuAmps system developed by NeuroScan as the brainwave system. Based on the International 10-20 system, we configured the EEG electrodes and captured the data signals. Before data were recorded, the impedance values of all electrodes were verified to be less than $5 \mathrm{k}$. The low- and high-frequency wave filters of the EEG signals were $0.05 \mathrm{~Hz}$ and $50 \mathrm{~Hz}$, respectively, and the sampling frequency was $1000 \mathrm{~Hz}$. Subsequently, we calculated the power of the four EEG frequency bands (delta, theta, alpha, and beta rhythm) at the 30 EEG electrode positions and the pairing coherence between the positions of the $30 * 30$ electrodes.

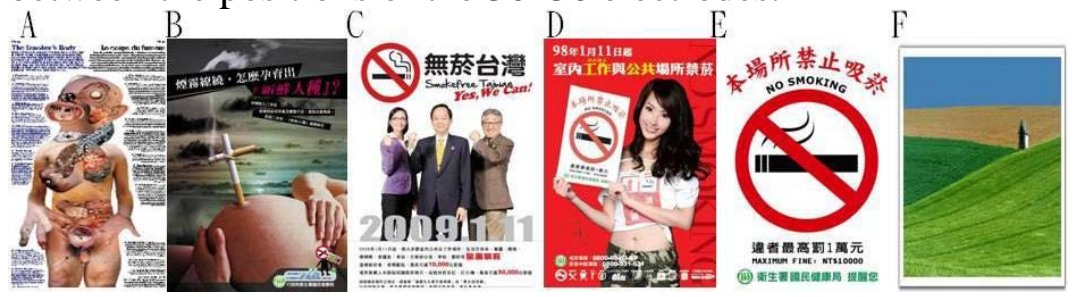

Fig.1. Five warning poster and one landscape image.

\subsection{Statistical Analysis}

The data analysis instruments used in this study were the Statistical Package for the Social Sciences 19.0 and Excel 2010. In the test of significance, the p value was set to .05. Our statistical methods are separately described as follows: (a) Descriptive statistics: This method was used for calculating the means and standard deviations of the power and coherence data in various frequency bands and films. (b) One-way analysis of variance (ANOVA): This method was used for determining the variations in the power and coherence data of the four wavebands when the participants viewed the different images. (c) General linear model and one-way ANOVA: This method was used for comparing the variations in the power and coherence data when the participants viewed the five labels and the neutral image (landscape).

\section{Results}

Table 1 shows the detailed EEG power distribution on sub-band EEG rhythms for the six images. According to the table, significant differences among the six images were observed, even in the overall EEG frequency range or specific EEG rhythms. When the participants viewed the image of the diseased organs, the power values obtained from the theta, beta, and overall (total power) frequency bands were the lowest among the various images. When the participants viewed the image of the pregnant woman, the power of the delta frequency band was the lowest, followed by the beta frequency band. When the participants viewed the image of the political and public figures, the power of the alpha frequency band was the lowest. By contrast, when the participants viewed the image of the young idol, the power values measured from the delta, theta, alpha, and overall frequency bands were the highest. 
Table.1. EEG power distribution of the six images. Data is represented as mean (sd)

\begin{tabular}{|c|c|c|c|c|c|c|c|c|}
\hline & $\begin{array}{c}\text { Diseased } \\
\text { organs }\end{array}$ & $\begin{array}{c}\text { Pregnant } \\
\text { woman }\end{array}$ & $\begin{array}{c}\text { Political and } \\
\text { public }\end{array}$ & Young idol & $\begin{array}{c}\text { Basic textual } \\
\text { declaration }\end{array}$ & Landscape & $\begin{array}{c}\text { ANOVA } \\
\text { F test }\end{array}$ & significance \\
\hline Total power & $6.49(7.48)$ & $6.82(8.32)$ & $7.62(10.9)$ & $7.92(12.5)$ & $7.34(9.46)$ & $7.11(8.94)$ & 9.35 & $.00^{* * * *}$ \\
\hline Delta power & $14.46(9.76)$ & $14.30(9.47)$ & $16.29(15.86)$ & $16.74(19.45)$ & $16.16(13.09)$ & $15.83(12.77)$ & 6.17 & $.00^{* * *}$ \\
\hline Theta power & $5.49(2.99)$ & $5.95(3.11)$ & $5.9(3.88)$ & $6.68(6.67)$ & $5.77(3.64)$ & $5.96(3.35)$ & 17.99 & $.00^{* * * *}$ \\
\hline Alpha power & $4.78(4.87)$ & $5.77(9.39)$ & $4.26(3.91)$ & $6.85(8.88)$ & $6.08(7.43)$ & $5.26(5.55)$ & 24.15 & $.00^{* * *}$ \\
\hline Beta power & $1.22(0.87)$ & $1.29(0.96)$ & $2.59(8.90)$ & $1.41(1.15)$ & $1.36(0.98)$ & $1.38(1.02)$ & 17.55 & $.00^{* * *}$ \\
\hline
\end{tabular}

Figure 2 shows the power topographies organized from the average EEG power responses from the 31 participants when viewing the six pictures.

\begin{tabular}{|c|c|c|c|c|c|c|}
\hline $\begin{array}{l}\text { Frequency } \\
\text { figure }\end{array}$ & $\begin{array}{c}\text { Diseased } \\
\text { organs }\end{array}$ & $\begin{array}{l}\text { Pregnant } \\
\text { woman }\end{array}$ & $\begin{array}{l}\text { Political and } \\
\text { public figure }\end{array}$ & Young idol & $\begin{array}{c}\text { Basic textual } \\
\text { declaration }\end{array}$ & Landscape \\
\hline Delta & & & & & & \\
\hline Theta & & & & & & \\
\hline Alpha & & & & & & \\
\hline beta & & & & & & \\
\hline All & & & & & & \\
\hline
\end{tabular}

Fig.2. EEG power topography on the six images.

Subsequently, we used multiple testing to compare the significance of each image with the landscape image. The t test results based on total power and sub-band power is listed in Table 2 .

Table.2. In the Dunnett t test, the groups were the controls with which all of the other groups were compared.

\begin{tabular}{|c|c|c|c|c|c|}
\hline & Diseased organs & Pregnant woman & Political and public figures & Young idol & Basic textual declaration \\
\hline Total power & $0.01(-)$ & 0.42 & 0.89 & $0.00(+)$ & 0.61 \\
\hline Delta power & 0.11 & 0.06 & 0.92 & 0.45 & 0.98 \\
\hline Theta power & $0.04(-)$ & 1.00 & 1.00 & $0.00(+)$ & 0.75 \\
\hline Alpha power & 0.41 & 0.36 & $0.01(-)$ & $0.00(+)$ & $0.04(+)$ \\
\hline Beta power & 0.84 & 0.98 & $0.00(+)$ & 1.00 & 1.00 \\
\hline
\end{tabular}

In addition to the responses triggered using the images of the pregnant woman, the other four pictures induced significantly different responses in the theta, alpha, and beta rhythm data of the participants. In contrast to viewing the neutral landscape image, the total and theta brainwave power of the participants viewing the diseased organs decreased. When viewing the image of the political and public figures, the alpha and beta brainwave power decreased in the participants. When viewing the image of the young idol, the total, theta, and alpha brainwave power values of the participants were higher than those when the participants viewed the landscape image. When the participants viewed the basic textual declaration, their alpha brainwave power was higher than that when viewing the landscape image. These were the results that differed significantly when compared with the respective brainwaves of the participants when viewing the landscape image. These results indicated that when the participants viewed these images, their response brainwave power varied. These results were compared with those shown in Figure 3 to analyze the relationship between the total brainwave power in the central, left, and right brain hemispheres of the participants. The results showed that the brainwave power of the participants in the central and left brain hemispheres differed significantly when viewing the image of the young idol $(p=.03$ and $p=.02)$. The results are presented in Table 3. 
Table.3. Comparison of the total brainwave power in the central, right, and left brain hemispheres when the landscape image was used as the control image.

\begin{tabular}{|c|c|c|c|c|c|}
\hline & Diseased organs & Pregnant woman & Political and public figures & Young idol & Basic textual declaration \\
\hline Central brain & 0.25 & 1.00 & 0.81 & 0.03 & 0.64 \\
\hline Right brain & 0.07 & 0.96 & 0.99 & 0.12 & 0.95 \\
\hline Left brain & 0.32 & 0.37 & 1.00 & 0.02 & 0.96 \\
\hline
\end{tabular}

In the Dunnett t test, the groups were regarded as the control groups and were compared with all of the other groups. When the levels of significance were higher than .05 , the mean values differed significantly.

Table.4. Channel pairs yielding significant statistical differences in the coherence comparison

between the responses of the five smoking cessation images and the neutral landscape image.

\begin{tabular}{|c|c|c|c|c|c|}
\hline Channel pairs & Diseased organs & Pregnant woman & Political and public figures & Young idol & Basic textual declaration \\
\hline $\begin{array}{l}\text { Frontal Lobe - } \\
\text { Frontal Lobe }\end{array}$ & $\begin{array}{l}\text { Fp1-[Fp2, F8, FT8]; } \\
\text { Fp2-[F8, F7, FC3, FT7]; } \\
\text { F3-[F8, FT8]; F4-F7; } \\
\text { Fz-[F8, F7, FT8]; } \\
\text { F8-[F7, FC3, FCz, } \\
\text { FT7]; F7-[FC4, FT8]; } \\
\text { FC3-FT8; }\end{array}$ & $\begin{array}{l}\text { Fp1-[F8, FT8]; } \\
\text { Fp2-F8; F3-[F8, } \\
\text { FT8]; F4-[F8, } \\
\text { FT8]; Fz-[F8, } \\
\text { FT8]; F8-[F7, } \\
\text { FC3]; FC4-FT8; } \\
\text { FC3-FT8; }\end{array}$ & $\begin{array}{l}\text { Fp1-[F8, FT8]; Fp2-FT7; } \\
\text { F3-FT8; F4-FT8; Fz-[F7, } \\
\text { FT7, FT8]; F8-FC3; } \\
\text { FC4-FT7; FC3-[FT7, FT8, } \\
\text { TP8]; FCz-FT7; }\end{array}$ & $\begin{array}{l}\text { Fp1-[Fp2, F4]; } \\
\text { F8-[F7, FC3]; } \\
\text { F8-FT7; F7-[FC4, } \\
\text { FT8]; FC4-FT7; } \\
\text { FC3-FT8; } \\
\text { FCz-FT7; }\end{array}$ & $\begin{array}{l}\text { Fp1-[F8, FT8]; Fp2-F8; } \\
\text { F3-[F8, FT8]; Fz-F8; } \\
\text { F8-[F7, FC3, FT7]; } \\
\text { F7-[FC4, FT8]; FC4-FT7; } \\
\text { FC3-[FT7,FT8]; }\end{array}$ \\
\hline $\begin{array}{l}\text { Frontal Lobe - } \\
\text { Parietal Lobe }\end{array}$ & $\begin{array}{l}\text { Fp1-CPz; F8-[C3, Cz, } \\
\text { CP3]; F7-[C4, CP4, } \\
\text { CPz]; FT7-T4; }\end{array}$ & $\begin{array}{l}\text { Fp1-C4; F8-C3; } \\
\text { FC4-T4; } \\
\text { FC3-[C4,T4,TP8]; }\end{array}$ & $\begin{array}{l}\text { Fp1-[C4,T4,CPz]; F8-CP3; } \\
\text { FC3-T4; FT7-[C4, Cz, T4, } \\
\text { CPz]; FT8-[C3, TP7]; }\end{array}$ & $\begin{array}{l}\text { F8-[C3, CP3]; } \\
\text { F7-[C4,Cz,T4,CPz]; } \\
\text { FC3-T4; FT7-[C4, } \\
\text { Cz, T4, CP4, CP3, } \\
\text { CPz]; FT8-T3; }\end{array}$ & $\begin{array}{l}\text { F8-C3; F7-T4; FC3-T4; } \\
\text { FT7-C4; FT8-T3; }\end{array}$ \\
\hline $\begin{array}{l}\text { Frontal Lobe - } \\
\text { Occipital Lobe }\end{array}$ & & & $\begin{array}{l}\text { Fp1-Oz; F4-O1; Fz-[O1, } \\
\text { Oz, T5]; FC4-O1; FCz-[T5, } \\
\text { O1]; FT7-[O1, Oz, O2]; } \\
\text { FT8-[O1, O2, Oz]; }\end{array}$ & FT7-Pz; & \\
\hline $\begin{array}{l}\text { Parietal Lobe - } \\
\text { Parietal Lobe }\end{array}$ & T4-[C3, CP3]; & & $\begin{array}{l}\text { C4-TP7; Cz-TP7; T4-[T3, } \\
\text { CP3, TP7]; CPz-[TP7, TP8]; }\end{array}$ & & \\
\hline $\begin{array}{l}\text { Parietal Lobe - } \\
\text { Occipital Lobe }\end{array}$ & & & $\begin{array}{l}\text { C3-O2; T4-[P3, T5, O1, O2, } \\
\text { Oz]; CPz-T5; TP8-Oz; }\end{array}$ & CPz-T5; & \\
\hline
\end{tabular}

We subsequently compiled the comparison of coherence channel pairs to differentiate participant responses between the five warning labels from the neutral landscape image. Table 4 lists the number of channel pairings yielding significant statistic differences. In identical channel pairing, the values of the coherence response to the landscape image were the lowest when compared with those of the other four images. The responses from the images of the diseased organs, pregnant woman, political and public figures, young idol, and basic textual declaration consisted of 30, 20, 53, 27, and 20 significant channel pairings, respectively. Regarding the EEG power distribution, the responses from the images of the diseased organs, pregnant woman, young idol, and basic textual declaration yielded significant channels in the regions of the frontal lobe to frontal lobe and frontal lobe to parietal lobe. The responses from the political and public figures yielded the largest number of significant channels, which were widely distributed in the regions of the frontal lobe to frontal lobe, frontal lobe to parietal lobe, frontal lobe to occipital lobe, parietal lobe to parietal lobe, and parietal lobe to occipital lobe.

\section{Discussion}

Numerous studies have examined the influence of viewing pictures on EEG variations. In this paper, we discuss the responses of the participants to viewing cigarette warning labels. In addition, we adopted the power and coherence EEG parameters, both of which effectively showed that different smoking cessation images prompted different smoker responses. Among our data, the variations in channel numbers and channel pair numbers caused by coherence responses were higher than those derived by power responses. The five warning labels provided different methods of appeal to the smokers.

However, no relationship between the characteristics of these differences and the smokers who ceased smoking was determined. We did not have a control group to verify the influence of smoking on the cognitive responses to various emotional images or on the brain structure, which subsequently influences the responses to the images viewed. However, relevant studies are available and have shown that smoking alters EEG waves or event-related potentials [17-18].

In addition, although EEG parameters changed, the types of emotional appeal on warning labels 
that can effectively facilitate subsequent smoking cessation remain unclear. Specifically, we do not have a definite conclusion on the degree of association between the types of emotional appeal and the subsequent strengthening of smoking cessation behavior. Thus, long-term follow-up and further discussion are required.

\section{Conclusion}

In this study, we established a method of using brain science results to examine the various possible effects of graphical cigarette warning designs and provided brain science data as a reference for following relevant designs.

\section{Acknowledgement}

This work has been supported by the Ministry of Science and Technology of Taiwan (Grants Nos. MOST 105-2221-E-468 -013). There is no any Conflict of Interest.

\section{References}

[1] Colin D Mathers , Dejan Loncar. Projections of global mortality and burden of disease from 2002 to 2030 . PLoS medicine 20063 (11) e442.

[2] Wen C-P, Levy D-T, Cheng T-Y, Hsu C-C, Tsai S-P. Smoking behavior in Taiwan, 2001. Tobacco control 200514 Suppl 1 i51-55.

[3] Chung Chih-wen, Wang Jung-der, Yu Cheng-fen, Yang Ming-chin. Lifetime medical expenditure and life expectancy lost attributable to smoking through major smoking related diseases in Taiwan. Tobacco control 200716 (6) 394-399.

[4] Bureau of Health Promotion. Taiwan Tobacco Control Annual Report 2010. Department of health, Taiwan 2010.

[5] Schinke, Steven Paul, and Lewayne D. Gilchrist. Preventing tobacco use among young people. Health \& social work 1986 11(1) 59-65.

[6] Chang Fong-ching, Chung Chi-hui, Yu Po-tswen, Chao Kun-yu. The impact of graphic cigarette warning labels and smoke-free law on health awareness and thoughts of quitting in Taiwan. Health education research 201126 (2) 179-191.

[7] World Health Organization. Showing the truth, saving lives: the case for pictorial health warnings. Geneva: World Health Organization 2009.

[8] David Hammond, Geoffrey Fong, A McNeill, Cummings K Michael. Effectiveness of cigarette warning labels in informing smokers about the risks of smoking: findings from the International Tobacco Control (ITC) Four Country Survey. Tobacco control 200615 Suppl 3 iii19-25.

[9] Judy Li, Grigg Michele. New Zealand: new graphic warnings encourage registrations with the quitline. Tobacco control 200918 (1) 72.

[10] Lalla Ilhame Sabbane, Tina M Lowrey, Jean-Charles Chebat. The Effectiveness of Cigarette Warning Label Threats on Nonsmoking Adolescents. Journal of Consumer Affairs 200943 (2) 332-345.

[11] Constantine I Vardavas, Gregory Connolly, Kostas Karamanolis, Anthony Kafatos. Adolescents perceived effectiveness of the proposed European graphic tobacco warning labels. European journal of public health 200919 (2) 212-217.

[12] Qing Zhang, Minho Lee. Analyzing the dynamics of emotional scene sequence using recurrent neuro-fuzzy network. Cognitive neurodynamics 20137 (1) 47-57.

[13] Rafael A Calvo, Sidney D'Mello. Affect Detection: An Interdisciplinary Review of Models, Methods, and Their Applications. IEEE Transactions on Affective Computing 20101 (1) 18-37.

[14] Marianne Littel, Ingmar H A Franken. Intentional modulation of the late positive potential in response to smoking cues by cognitive strategies in smokers. PloS one 20116 (11) e27519.

[15] Robert E Featherstone, Jennifer M Phillips, Tony Thieu, Richard S Ehrlichman, Tobias B Halene, Steven C Leiser, Edward Christian, Edwin Johnson, Caryn Lerman, Steven J Siegel. 
Nicotine receptor subtype-specific effects on auditory evoked oscillations and potentials. PloS one 20127 (7) e39775.

[16] Maartje Luijten, Marianne Littel, Ingmar H. A. Franken. Deficits in inhibitory control in smokers during a Go/NoGo task: an investigation using event-related brain potentials. PloS one 20116 (4) e18898.

[17] Edward F Dominoa, Lisong Nia, Michael Thompsona, Huilei Zhanga, Hiroki Shikatab, Hiromi Fukaib, Takeshi Sakakib, Ippei Ohyab. Tobacco smoking produces widespread dominant brain wave alpha frequency increases. International journal of psychophysiology 200974 (3) 192-198.

[18]H N Alexander Logemann, Koen Böcker, P K H Deschamps, J L Kenemans. The effect of the augmentation of cholinergic neurotransmission by nicotine on EEG indices of visuospatial attention. Behavioural brain research 2014260 67-73. 